\title{
Espacios de trabajo geométrico sintético y analítico de profesores y su práctica en el aula
}

\section{Synthetic and analytic geometrical working space of teachers and their practices in the classroom}

Carolina Henríquez Rivas, Elizabeth Montoya Delgadillo

Instituto de Matemáticas. Pontificia Universidad Católica de Valparaiso, Chile

carohenriquezrivas@gmail.com, emontoya@ucv.cl

RESUMEN • En este trabajo mostramos los resultados de un estudio realizado sobre el trabajo matemático de dos profesores nóveles en su práctica en el aula, cuando las temáticas involucran los enfoques de geometría euclidiana sintético y analítico. Presentamos el espacio de trabajo matemático (ETM) idóneo de cada docente, específicamente las circulaciones entre las componentes de los planos epistemológico y cognitivo, y el paradigma geométrico privilegiado. Los resultados proporcionan datos empíricos respecto a la práctica docente al usar distintos métodos geométricos y sobre la posibilidad de complementarlos; además, el tema se presenta como una nueva dimensión de estudio para el ETM.

PALABRAS CLAVE: trabajo matemático; paradigma geométrico; profesor.

ABSTRACT - In this paper we show the results of a study in relation to the mathematical work and practice of two novice teachers, when the classroom topic involved the differing views related to synthetic and analytic Euclidean geometry. We present the ideal mathematical working space (MWS) for each teacher, specifically, the movements between the components of the epistemological and cognitive planes, and the privileged geometric paradigm. The results provide empirical information regarding the teaching practice when using different geometric methods and on the possibility of complementing them, and furthermore the topic is presented as a new way of studying through the MWS.

KEYWORDS: mathematical working; geometrical paradigm; teacher.

Fecha de recepción: enero 2014• Aceptado: febrero 2015

Henríquez, C., Montoya, E., (2015) Espacios de trabajo geométrico sintético y analítico

de profesores y su práctica en el aula. Enseñanza de las Ciencias, 33.2, pp. 51-70 


\section{INTRODUCCIÓN}

En este artículo se hace referencia a una problemática vigente relacionada con la enseñanza de la geometría en el nivel secundario; la complementariedad entre los enfoques de geometría euclidiana con y sin coordenadas cartesianas -usamos la denominación geometría analítica y geometría sintética-. Para diferenciar entre tales enfoques, nos basamos en la distinción que ofrece Klein (1908): en la geometría sintética se estudian las figuras en sí mismas sin intervención de fórmulas y en la analítica estas se aplican mediante el uso de los sistemas de coordenadas. Según Klein «... la diferencia entre ambas especies de Geometría es puramente cuantitativa: según predominen las fórmulas o las figuras, se tiene una u otra Geometría» (p. 74).

En el célebre Programa de Erlangen, Klein preconiza el cese a las disputas entre las geometrías sintética y analítica, pues bajo este punto de vista una "geometría» vendría a ser el conjunto de propiedades invariantes mediante las transformaciones del grupo correspondiente (Bourbaki, 1969).

Desde la perspectiva de la enseñanza de la geometría, se trata de un asunto que ha sido abordado desde hace más de un siglo por diversos autores, suscitando controversias que cada cierto tiempo han sido replanteadas. A comienzos del siglo XX, Klein señala acerca de lo sobrecargada que se encuentra la enseñanza y expresa las exigencias que debe satisfacer un libro de texto, por ejemplo, debe tomar en cuenta el punto de vista psicológico del sujeto a quien se enseńa dependiendo de su edad (Klein, 1908). Según Klein, la enseñanza de la geometría debe considerar, entre otras, la práctica del profesor, la fusión de diversas ramas de la Matemática, conceder importancia al dibujo y la representación de las figuras geométricas, e introducir antes el concepto de función en geometría analítica.

A partir de la década de los sesenta, las nuevas tendencias de la educación matemática y sus implicancias en la enseñanza de la geometría provocaron debate. En el prólogo del libro Álgebra lineal y geometría elemental, Dieudonné (1964) manifiesta su rechazo a la separación en la enseñanza tradicional de las que llama pseudociencias, como la geometría pura, la geometría analítica y trigonometría (entre otras). Dieudonné sostiene que se olvide su existencia e incluso hasta sus nombres, en favor de las ventajas que proporciona el álgebra lineal.

En el año 1973, la Unesco reporta sobre la falta de consensos y la necesidad de realizar estudios pedagógicos sobre la enseñanza de la geometría, especialmente en la escuela secundaria. En este marco, el estudio de Bishop (1987) describe algunos obstáculos para el aprendizaje de la geometría; como los obstáculos para el aprendizaje del espacio, puesto que las concepciones geométricas están inspiradas en el formalismo en lugar del empirismo e intuicionismo. Asimismo, otro tipo de obstáculos se dan en el caso de las geometrías vectoriales y analíticas, advirtiendo que corren el riesgo de volverse rápidamente algebraicas.

En relación con la enseñanza de la geometría en el nivel secundario, Santaló (1980) se refiere a la urgencia de establecer nuevos currículos, considerando las capacidades de aprendizaje de los estudiantes. El autor expone que mantener las exigencias de rigor y coherencia para cualquier edad y cualquier alumno no es posible, y sostiene «no es malo que los estudiantes conozcan distintos métodos y distintos puntos de partida» (p. 26). Para Santaló, esto se trata de una confusión entre la matemática como materia de investigación de matemáticos profesionales y la matemática como disciplina formativa que debe ser enseñada a estudiantes.

Sobre los intentos por unificar la tradicional geometría sintética con la geometría analítica, De Villiers (1993) resalta el poder de las transformaciones geométricas en la matemática escolar. En otras palabras, las transformaciones geométricas no solo en tópicos de geometría, sino su relación con el álgebra y la trigonometría. De Villiers concluye que la enseñanza de la geometría puede ampliarse y enriquecerse con las transformaciones geométricas y su aplicación en diversas áreas del currículo. 
Desde la perspectiva cognitiva desarrollada por Duval (1995, 1999 y 2005), se destacan los sistemas semióticos con registros de representación y tratamientos específicos, lo que induce a considerar que en ambos enfoques, sintético y analítico, existe una manera específica de trabajar. Particularmente, el proceso de visualización que opera en geometría, no solo requiere de las figuras geométricas o los gráficos cartesianos, sino que un trabajo semiótico asociado a la identificación, tratamientos y conversiones, en coordinación con razonamientos discursivos.

El trabajo de Gascón (2002 y 2003) es considerado como una aproximación a nuestra problemática de investigación. Gascón propone a través de cierto tipo de problemas, una forma de conectar el enfoque sintético con el analítico a fin de poner de manifiesto su complementariedad. Sin embargo, pensamos que esto no es suficiente para abordar la problemática en profundidad; los aspectos que nos interesa investigar contemplan el nivel de enseñanza y las condiciones que permiten a un individuo (investigador, profesor o estudiante) efectuar su espacio de trabajo geométrico, para estudiar y aportar evidencia empírica sobre la actividad matemática.

Respecto al estudio de la actividad matemática en el aula, especialmente centrado en la práctica del profesor, el trabajo de Badillo, Figueiras, Font y Martínez (2013) muestra la complejidad matemática del análisis durante el desarrollo de una clase, a través del diseño y la utilización de un instrumento para su estudio basado en el enfoque ontosemiótico, el cual permitió desarrollar un análisis comparativo de la práctica matemática en el aula de tres profesores.

Para profundizar en el trabajo matemático de profesores en su práctica en el aula con un modelo teórico específico, y particularmente sobre temáticas de geometría sintética y analítica, hemos considerado la teoría Espacio de Trabajo Matemático (Kuzniak, 2011). Nos interesa conocer cómo se ponen de manifiesto aspectos matemáticos y cognitivos en la práctica del profesor de secundaria, cuando las temáticas se desarrollan según cada enfoque geométrico.

En esta investigación se deben precisar las nociones de tránsito y complementariedad: el tránsito es entendido como el paso del enfoque sintético al analítico (o viceversa); la complementariedad es relativa a que estos se influencien mutuamente. Esto favorece por un lado, profundizar en contenidos que se estudian en la escuela, y por otro, la posibilidad de conectar la geometría con otros dominios de la matemática.

En el siguiente apartado se presenta una breve descripción sobre dos dispositivos que son un referente para el trabajo del profesor y su formación inicial, relativos a la geometría en la educación secundaria (o educación media).

\section{HÁBITAT DE LA GEOMETRÍA EN LA EDUCACIÓN MEDIA}

Sobre los dispositivos de carácter oficial que son un referente para el quehacer del docente en Chile, se han considerado dos documentos: el Marco Curricular y los Estándares Orientadores para Carreras de Pedagogía en Educación Media. ${ }^{1}$

En el Marco Curricular (Ministerio de Educación [MINEDUC], 2009), particularmente en el eje geometría, se establece «ampliar la base epistemológica de la geometría, mediante las trasformaciones rígidas en el plano, los vectores y la geometría cartesiana» (p. 146). Según señala el currículo, esto se manifiesta en el esfuerzo por incluir diferentes enfoques para el tratamiento de los problemas, relacionar el eje de geometría con los otros ejes: números, álgebra, datos y azar.

De modo general en el currículo, el eje de geometría presenta:

1. Instrumento desarrollado como un referente para las instituciones formadoras de profesores de educación media. 
- Hasta octavo básico (13 años), el trabajo geométrico se efectúa sin el uso de coordenadas, donde predominan los tratamientos figurales, el estudio de propiedades, construcciones y transformaciones de figuras.

- En primero medio (14 años), se introduce el plano cartesiano para representar puntos, figuras, vectores y las transformaciones isométricas. Además, incluye la congruencia de figuras planas, sin explicitar en qué enfoque geométrico trabajar.

- En segundo medio (15 años), el trabajo no considera el uso de las coordenadas, se basa en la semejanza de figuras planas, ángulos en la circunferencia y la demostración de algunos teoremas relacionados con dichas temáticas. Hasta este nivel el tema de la geometría sintética, analítica, su complementariedad y el paso de un enfoque a otro es invisible para el docente.

- En tercero medio (16 años), la relación entre los enfoques geométricos aparece explícitamente en uno de los objetivos fundamentales «Comprender la geometría cartesiana como un modelo para el tratamiento algebraico de los elementos y relaciones entre figuras geométricas» (p. 188). Sin embargo, no se aporta información que oriente el trabajo del docente, pues el énfasis, según lo declarado, está en los tratamientos algebraicos, dejando de igual forma aspectos que no son considerados ni cuestionados. ${ }^{2}$

En los Estándares Orientadores para Carreras de Pedagogía en Educación Media (Centro de Perfeccionamiento, Experimentación e Investigaciones Pedagógicas [CPEIP], 2012), el tema se presenta en el área geometría, especialmente en los estándares 12 y 14, y en sus descriptores, en los cuales se exponen las capacidades que debe poseer el futuro profesor, como promover la visualización y argumentación de los estudiantes. Además, el documento expresa la intención por comparar ambos enfoques, pero no dice cómo hacerlo. Al respecto, uno de los descriptores dice: "Resuelve problemas de geometría euclidiana con y sin coordenadas cartesianas, comparando ambos enfoques» (p. 119). Sin embargo, esta información no es suficiente para orientar o favorecer el tránsito y complementariedad entre los enfoques geométricos.

La problemática hasta aquí presentada, si bien se declara, no presenta ejemplos que orienten cómo proceder con relación al tránsito y complementariedad entre los enfoques, ni cómo enseñarlos. A lo cual se suma la falta de evidencias empíricas que permitan dar cuenta de lo que efectivamente realiza un profesor en su práctica en el aula. Cabe preguntarse entonces ¿cómo abordará esta temática un profesor en su práctica en el aula? Y por otro lado, ¿qué perspectivas se pueden ofrecer para abordar esta problemática?

Presentamos un análisis cualitativo al trabajo en el aula de docentes nóveles sustentado en una teoría particularmente apropiada para estudiar la actividad matemática del profesor, llamada Espacio de Trabajo Matemático, la cual se describe a continuación.

\section{MARCO TEÓRICO}

Este estudio se sustenta principalmente en la teoría Paradigmas Geométricos y Espacio de Trabajo Geométrico desarrollada inicialmente por de Houdement y Kuzniak (1996, 1999 y 2006). En la actualidad es llamada Espacio de Trabajo Matemático (ETM), que surge con la necesidad de profundizar en otros dominios de la matemática (Kuzniak, 2011).

2. En el último nivel de la enseñanza obligatoria chilena, cuarto medio, se estudian las primeras nociones de geometría analítica del espacio. El paso del plano al espacio no será abordado. 


\section{Espacio de Trabajo Matemático}

El ETM se define como un ambiente organizado para permitir el trabajo de las personas que resuelven tareas matemáticas. El ETM se constituye por dos planos, cognitivo y epistemológico, en relación directa con los contenidos matemáticos del dominio en juego. En cada plano hay tres componentes; en el cognitivo están presentes los procesos de visualización, construcción y prueba, y en el epistemológico, representante (o «representamen»), artefactos y referencial. Las componentes de los planos se articulan mediante tres génesis: semiótica, instrumental y discursiva. La génesis semiótica está asociada a las representaciones de los objetos matemáticos. La génesis instrumental permite hacer operatorios los artefactos en el proceso constructivo. Por último, la génesis discursiva que da sentido al referencial (definiciones, propiedades) para ponerlo al servicio del razonamiento matemático (Kuzniak, 2011). En la figura 1 se muestra el ETM y las génesis que permiten articular los planos.

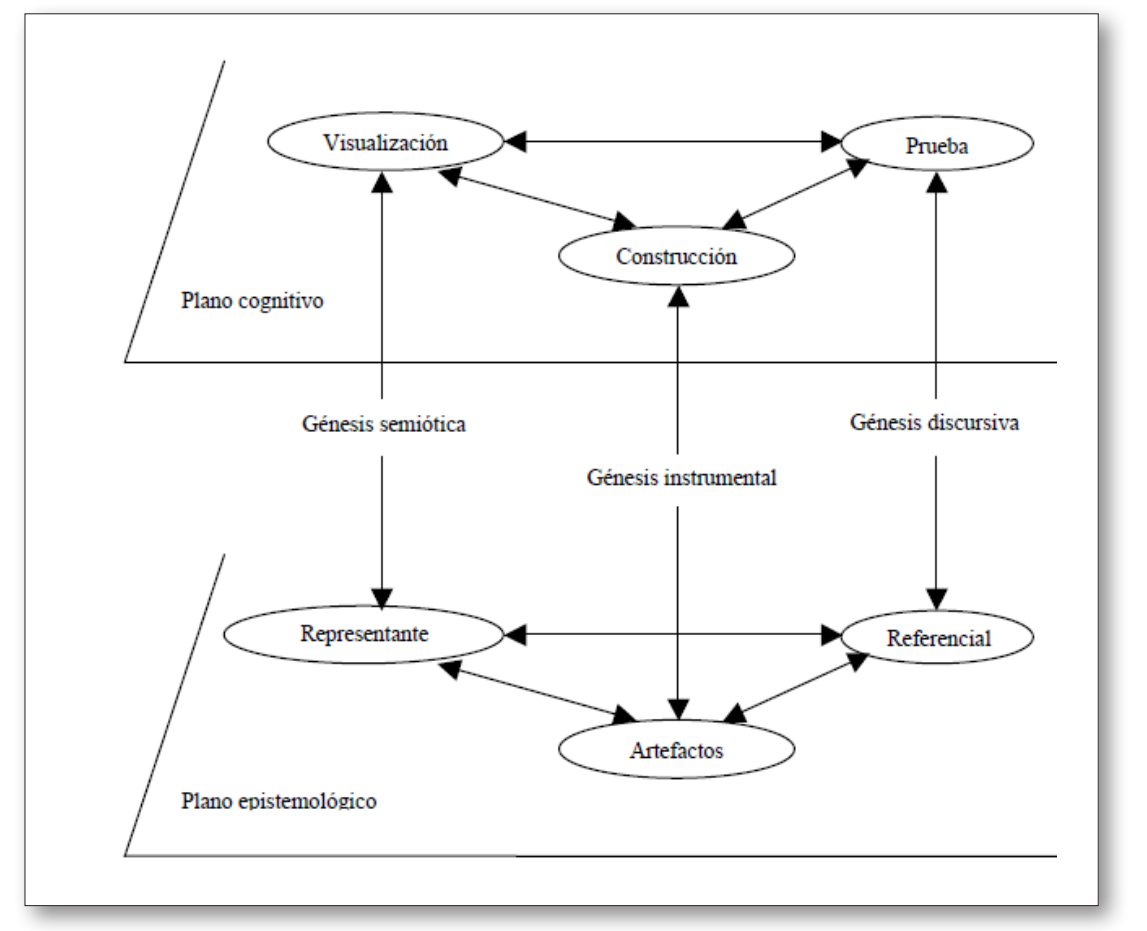

Fig. 1. Espacio de trabajo matemático y sus génesis (Kuzniak, 2011).

La idea del representante (representamen) está relacionada a la noción signo de Peirce (1978), y tiene que ver con el objeto matemático bajo formas más o menos abstractas: íconos, índices y símbolos. Un signo remite a su objeto de alguna de estas tres formas, según un proceso semiótico -llamado aquí visualización - involucrado en función de las significaciones de su utilizador. La articulación entre ambas componentes se produce con la génesis semiótica, relacionando representante y visualización. Esta génesis se sustenta en la concepción cognitiva de Duval (1995), quien toma en cuenta la operación de conversión de un sistema semiótico a otro y los tratamientos al interior de un registro.

En relación con los artefactos en el ETM, estos se vinculan con la concepción de Rabardel (1995), quien distingue que un artefacto puede ser material o un sistema simbólico empleado como un medio para la acción. El instrumento es considerado como una entidad mediadora entre el sujeto y el objeto sobre el cual se dirige la acción y, además, una entidad mixta que comprende el artefacto y componentes cognitivos relacionados con los esquemas de uso. En la concepción del ETM, el proceso de cons- 
trucción está determinado por los instrumentos utilizados. La articulación entre ambas componentes se produce con la génesis instrumental (Artigue, 2002; Trouche, 2002), relacionando artefactos con el proceso de construcción.

La componente referencial remite a los elementos de índole teórico matemático del dominio en juego (geometría, álgebra, análisis, etc.). Aquí se encuentra el hábitat matemático del objeto, es decir, propiedades y definiciones involucradas en un razonamiento y cómo estas son utilizadas en un discurso para argumentar y convencer -llamado aquí prueba-. La génesis discursiva articula el proceso de prueba con el referencial.

En los distintos ETM es posible identificar tres tipos, dependiendo de la función de la reflexión del sujeto cuando se enfrenta a un problema determinado: uno referencial, definido de manera ideal sobre criterios matemáticos (ETM de referencia); un espacio definido en términos de enseñanza en una institución dada con una función definida (ETM idóneo), y un espacio propio de cada utilizador fruto de la reflexión entre sus conocimientos matemáticos y los que utiliza para resolver una tarea (ETM personal). En esta investigación, los análisis están referidos al ETM idóneo del profesor.

Cabe señalar que hasta este momento, se han explicado aspectos del ETM en general. Para definir los elementos que constituyen el ETM en geometría, se deben considerar las particularidades del dominio.

\section{Espacio de Trabajo Geométrico $\left(\mathrm{ETM}_{\mathrm{G}}\right)$}

$\mathrm{Al}$ considerar las particularidades del dominio geométrico, la génesis semiótica se denomina génesis figural, y permite describir el proceso semiótico que está asociado al pensamiento visual que opera en geometría (Kuzniak, 2011). El espacio real y local se presenta como una componente particular del $\mathrm{ETM}_{\mathrm{G}}$ en el plano epistemológico (el representante del ETM). El aspecto real es el acceso a los objetos como el resultado de la abstracción del modelo, y el aspecto local concierne al trabajo con una parte del modelo.

Para analizar las componentes de los planos del $\mathrm{ETM}_{\mathrm{G}}$, se han incluido enfoques que lo complementan. Según Duval (1999), la visualización está basada en la producción de una representación semiótica (figuras geométricas, gráficos cartesianos) de un objeto. Según Duval (2005), se distinguen dos modos de visualizar que pueden funcionar según el tipo de operación con las figuras y cómo se movilizan sus propiedades; un modo icónico y otro no-icónico. Estos dos mecanismos de visualización se relacionan con cuatro formas de ver en función del rol de las figuras en las actividades geométricas propuestas a los alumnos: para el botaniste y arpenteur-géomètre, ${ }^{3}$ el reconocimiento de lo que representan las formas se hace por el parecido con el objeto (real) que representa, o en su defecto, por comparación con un modelo tipo de formas (una figura particular sirve de modelo y las otras figuras son reconocidas según su grado de parecido con este modelo); para el constructeur e inventeur-bricoleur, el trabajo implica la deconstrucción de las formas ya reconocidas visualmente (deconstrucción instrumental para construir una figura, la descomposición heurística, y la deconstrucción dimensional).

En la componente artefacto se considera la concepción (ya citada) de Rabardel (1995), el cual puede ser material, como una regla, un compás o un software, los cuales permiten trabajar sobre una figura. $\mathrm{O}$ bien, estos pueden ser simbólicos, que permiten producir un resultado en un registro semiótico distinto al figural.

La concepción de Balacheff sobre el proceso de prueba distingue entre las pragmáticas e intelectuales, y una tipología propia para cada una de estas pruebas que se diferencian por el estatus de los

3. En relación a las cuatro formas de visualización descritas por Duval (2005), hemos dejado las palabras en original, pues nos parece que su traducción al español no es la más apropiada. 
conocimientos en cuestión y la naturaleza de la justificación subyacente (Balacheff, 1987). Las pruebas pragmáticas (empirismo ingenuo, experiencia crucial, ejemplo genérico) son aquellas que recurren a la acción sobre los objetos y supone la posibilidad de tener acceso a la realización material de una tarea para justificar afirmaciones sobre ellos. Las pruebas intelectuales (experiencia mental, demostración, cálculo sobre el enunciado) son aquellas que recurren a la formulación de propiedades y relaciones entre los objetos en cuestión.

\section{Paradigmas geométricos}

Desde esta perspectiva podemos observar cómo un sujeto reflexiona de acuerdo con las creencias, técnicas y el conocimiento de distintos modelos geométricos cuando desarrolla una tarea específica, es decir, que el $\mathrm{ETM}_{\mathrm{G}}$ puede variar dependiendo del paradigma geométrico dominante.

Sobre la formulación de los Paradigmas Geométricos, Houdement y Kuzniak (1996, 1999, 2006) identifican tres tipos paradigmas que coexisten en la enseñanza de la geometría. En el paradigma geometría natural (GI), la geometría es sobre objetos reales y la importancia de la aproximación y de la medida, el trabajo es de tipo material, por tanto la fuente de validación es el mundo real y sensible. En el paradigma geometría axiomática natural (GII), los objetos geométricos son descritos por una propiedad, su definición, el razonamiento de validación se funda sobre las leyes hipotéticas deductivas del sistema axiomático en juego (que puede ser incompleto). El paradigma geometría axiomática formal (GIII) se caracteriza por la separación entre la axiomática y la realidad, donde los objetos provienen de una axiomática con toda la rigurosidad y formalismo del modelo geométrico elegido, el razonamiento de validación se realiza a través del sistema formal de axiomas del modelo subyacente.

Esta idea de paradigma geométrico ha sido inspirada por la noción de paradigma según Kuhn, de su trabajo La estructura de las revoluciones cientificas. En este sentido, un paradigma designa el conjunto de creencias, técnicas y valores que comparte un grupo científico, y el acceso al paradigma pasará por la resolución de un cierto número de problemas característicos (Kuzniak, 2011).

Por último, cabe señalar que es posible analizar diferentes entradas al ETM $\mathrm{G}_{\mathrm{G}}$ y ver cómo se articulan los planos epistemológico y cognitivo mediante las génesis, especificando las componentes puestas en juego; es lo que denominamos circulación entre las componentes de los planos en el ETM $\mathrm{E}_{\mathrm{G}}$. En lo que sigue, se analizan las circulaciones de dos espacios de trabajo geométricos, además, se identifica el paradigma geométrico privilegiado.

\section{DESCRIPCIÓN DEL ESTUDIO}

\section{Características generales y selección de clases}

Se propone un estudio de corte cualitativo sobre el trabajo en el aula de dos profesores nóveles ${ }^{4}$ (PD) de enseñanza media durante el segundo semestre de 2011 y primer semestre de 2012, grabando 10 (o más) sesiones de clases a 6 PD de 4 ciudades y formaciones iniciales diferentes, de universidades chilenas pertenecientes al Consejo de Rectores. ${ }^{5}$ En general, la formación exigida al profesor de secundaria en Chile es de cinco años, y contempla cursos de matemática, de educación matemática y prácticas profesionales -los énfasis varían según la institución formadora-.

4. Profesores con hasta tres años de ejercicio profesional. Nos focalizamos en estos profesores, ya que este trabajo se suscribe en una investigación más amplia sobre el ETM de profesores nóveles en Chile.

5. Organismo chileno integrado por los Rectores de las veinticinco universidades públicas y tradicionales. 
Los dos PD analizados fueron elegidos por los temas que trataron en sus respectivas sesiones de clases. Esto significa que los PD que se presentan y analizan en su práctica en el aula, fueron seleccionados para evidenciar el tratamiento que abordaron al enseñar tópicos de geometría con y sin coordenadas.

Para llevar a cabo el estudio se escogieron algunos métodos de investigación, como la observación de clases no participante, grabaciones y transcripciones de clases, el estudio al hábitat del problema en educación media y la revisión de antecedentes sobre el tema. Cabe señalar que los docentes participaron de forma voluntaria. Las sesiones observadas de ambos PD fueron desarrolladas en el horario habitual de la asignatura de matemática y su duración fue de 90 minutos. La información general de cada PD y el total de clases observadas se presenta en la tabla 1.

Tabla 1.

Profesores nóveles participantes

\begin{tabular}{|c|c|c|c|l|c|}
\hline Profesor & Género & $\begin{array}{c}\mathrm{N}^{\circ} \text { de clases } \\
\text { observadas }\end{array}$ & Nivel & Temas tratados & $\begin{array}{c}\text { Uso de tecnología } \\
\text { en las clases }\end{array}$ \\
\hline PD1 & $\mathrm{F}$ & 10 & $2^{\circ}$ Medio & $\begin{array}{l}\text { Semejanza de figuras planas, } \\
\text { productos notables, fracciones } \\
\text { algebraicas }\end{array}$ & No \\
\hline PD2 & M & 13 & $1^{\circ}$ Medio & $\begin{array}{l}\text { Transformaciones isométricas, } \\
\text { regularidades numéricas, ecuación } \\
\text { de primer grado }\end{array}$ & Sí \\
\hline
\end{tabular}

Posteriormente, para seleccionar las clases que fueron analizadas, se consideraron aquellas que trataban contenidos que curricularmente se encuentran en el eje geometría, pues las clases de álgebra no contemplaron temas donde aparece el sistema de coordenadas cartesianas. La sesión seleccionada de PD1 se refiere al Teorema de Thales, el cual corresponde a uno de los teoremas estudiados en secundaria en que se usan métodos sintéticos. La sesión escogida de PD2 es referida a Traslaciones en el plano, se trata de la primera sesión teórica y práctica sobre transformaciones isométricas en el plano cartesiano, las siguientes clases trataron sobre aplicación y composición de transformaciones isométricas. Asimismo, resultó relevante para este estudio que:

- PD1 trabaja en el enfoque geométrico sin coordenadas y PD2 con coordenadas cartesianas, además, las clases corresponden a niveles y temas curricularmente distintos.

- PD1 no utiliza tecnología y PD2 sí utiliza, lo cual permitió identificar cómo se emplearon los artefactos en el proceso de construcción, y sobre el rol de la visualización dinámica en el caso del uso de tecnología.

\section{Análisis de tareas en la clase}

La transcripción de las clases grabadas permitió identificar la totalidad de las tareas planteadas por cada PD durante cada clase. En relación con las tareas y su vínculo con el ETM, para Kuzniak (2011) «... los problemas no son parte del espacio de trabajo pero son su razón de ser y su activador» (p. 13). Por esta razón, al estudiar el ETM resulta fundamental identificar cuál es la tarea asociada a dicho trabajo. Sobre la noción que adoptaremos de tarea (o problema) concordamos con la concepción de Chevallard (1999), para quien una tarea ( $t$ ) se expresa por un verbo y supone un objeto relativamente preciso. Bajo esta concepción, las tareas ${ }^{6}$ son «construcciones institucionales, cuya reconstrucción en tal institución, y por ejemplo en tal clase, es un problema completo, que es el objeto mismo de

6. Incluidos tipos de tareas y géneros de tareas. En este trabajo, solo consideramos la noción de tarea. 
la didáctica» (p. 223). Para efectos del análisis, una vez identificadas las tareas que activan el ETM , distinguimos aquella que denominamos clave; se trata de la tarea propuesta en el episodio donde el docente focaliza la atención en el tema central de la clase. Con el objeto de organizar esta información, se elaboró la tabla 2, que nos ayudó a efectuar un análisis global sobre las tareas dadas por cada PD y seleccionar la $t$ clave que permitió analizar el $\mathrm{ETM}_{\mathrm{G}}$ idóneo en torno a una $t$ dada.

Tabla 2.

Tareas dadas por el PD durante la clase

\begin{tabular}{l|l|l} 
Tema central de la clase & Episodio de la clase & Tarea $(t)$
\end{tabular}

Para contextualizar cada clase y seleccionar la $t$ clave, la información fue organizada según dos aspectos:

1. Contextualización de la clase. Se revisó la relación entre el tema central de la clase, las tareas y el currículo vigente, se identificaron los conocimientos previos de los estudiantes y se efectuó la descripción del ambiente de la clase según aspectos del Marco para la buena enseñanza (CPEIP, 2008).

2. Tareas dadas por el PD durante la clase. Se organizaron las tareas según el episodio de la clase (tabla 2), y luego, se seleccionó la $t$ clave para analizar el $\mathrm{ETM}_{\mathrm{G}}$ idóneo del docente.

Una vez seleccionada la $t$ clave, para analizar el trabajo en el aula de cada docente se tomaron en cuenta los siguientes tres aspectos:

3. Descripción del trabajo desarrollado por cada PD en torno a la $t$ clave. Se presenta una descripción según el episodio de la clase, lo cual contribuyó en una correcta interpretación de los análisis desarrollados.

4. Análisis de la circulación de cada $\mathrm{ETM}_{\mathrm{G}}$ idóneo. Se caracterizó la circulación entre las componentes de los planos del $\mathrm{ETM}_{\mathrm{G}}$ y las génesis puestas en juego, en torno a la $t$ clave.

5. Análisis del paradigma geométrico privilegiado. Se analizó el trabajo en torno a elementos del modelo geométrico subyacente en la enseñanza.

Cabe señalar que se utilizará la notación $\mathrm{ETM}_{\mathrm{GS}}$ cuando el trabajo geométrico es sintético y $\mathrm{ETM}_{\mathrm{GC}}$ cuando es analítico.

\section{ANÁLISIS DE RESULTADOS}

\section{Primer caso: $\mathrm{ETM}_{\mathrm{GS}}$ idóneo de PD1}

\section{Contextualización de la clase}

La sesión se desarrolló en el año 2011, en la etapa final del año escolar. El nivel corresponde a segundo año medio (15 años). El tema central declarado por PD1 es el Teorema de Thales, el cual según el currículo (MINEDUC, 2009), se ubica en el eje geometría, específicamente, el Contenido Mínimo Obligatorio 12 que dice «Aplicación del teorema de Thales sobre trazos proporcionales...» (p. 187).

Sobre los conocimientos previos de los estudiantes, según lo declarado por PD1 al iniciar la clase, estos son reconocer triángulos semejantes usando teoremas de semejanza y aplicación del teorema de Pitágoras, considerado en el primer episodio de la clase cuando el docente propone la primera tarea $t_{1}$. 
En relación al ambiente de la clase, PD1 establece un clima de respeto con sus alumnos, un ambiente organizado de trabajo y proporciona oportunidades de participación. Sin embargo, contribuye en menor medida al desarrollo de la autonomía y a la indagación por parte de los estudiantes.

En la tabla 3 se muestran las tareas dadas por PD1 durante la clase y el episodio que caracteriza el instante de la tarea $(t)$.

Tabla 3.

Tareas dadas por PD1 y selección de $t$ clave

\begin{tabular}{|c|cl|l|}
\hline $\begin{array}{c}\text { Tema central de } \\
\text { la clase }\end{array}$ & \multicolumn{2}{|c|}{ Episodio de la clase } & \multicolumn{1}{c|}{ Tarea $(t)$} \\
\hline $\begin{array}{c}\text { Teorema de } \\
\text { Thales }\end{array}$ & 1. & $\begin{array}{l}\text { Recuerda } \\
\text { conocimientos previos }\end{array}$ & $\begin{array}{l}t_{1} \text { : Usar el teorema de Pitágoras para determinar la } \\
\text { diagonal de cuadrados de lado 1, 3 y 5 } u \text {. }\end{array}$ \\
\cline { 2 - 4 } & $\begin{array}{l}\text { 2. } \\
\text { Trata teorema de } \\
\text { Thales }\end{array}$ & $\begin{array}{l}t_{2}: \text { Calcular la medida del cateto de un triángulo } \\
\text { semejante a otro triángulo dado. }\end{array}$ \\
\cline { 2 - 4 } & 3. $\begin{array}{l}\text { Realiza ejercitación de } \\
\text { Teorema de Thales }\end{array}$ & $\begin{array}{l}t_{3}: \text { Calcular un valor desconocido dados triángulos } \\
\text { semejantes. }\end{array}$ \\
\cline { 2 - 4 } & 4. $\begin{array}{l}\text { Trata teorema de } \\
\text { Thales, caso general }\end{array}$ & $\begin{array}{l}t_{4}: \text { Calcular la medida de segmentos proporcionales } \\
\text { según una figura. }\end{array}$ \\
\cline { 2 - 4 } & 5. $\begin{array}{l}\text { Realiza ejercitación de } \\
\text { Teorema de Thales }\end{array}$ & $\begin{array}{l}t_{5}: \text { Calcular un valor desconocido entre tres o dos rectas } \\
\text { paralelas intersecadas por otras dos rectas. }\end{array}$ \\
\hline
\end{tabular}

En este caso, la $t$ clave seleccionada es «calcular la medida del cateto de un triángulo semejante a otro triángulo dado", pues se trata del episodio central de la clase, y es a partir del trabajo generado por $t_{2}$ que se realizan los siguientes tres episodios de la clase.

\section{Descripción del trabajo en torno a la t clave}

El trabajo es activado por $t_{2}$ : Calcular la medida del cateto de un triángulo semejante a otro triángulo dado. PD1 escribe el título «Teorema de Thales» e inicia el trabajo dando la correspondencia entre los catetos de dos triángulos dibujados en la pizarra. Declara que la altura de una pirámide y su sombra están en correspondencia con un bastón y su respectiva sombra, argumentando que son semejantes. Representa esta situación a mano alzada dibujando dos triángulos (imagen 1). Aquí PD1 no explica por qué la primera figura representada en configuración $2 \mathrm{D} / 2 \mathrm{D}^{7}$ correspondería a una configuración $3 \mathrm{D} / 2 \mathrm{D}$, situación cuestionada por un estudiante. El trabajo se focaliza en la visión de las figuras (Duval, 1999). Luego, PD1 recuerda la semejanza de triángulos, tema tratado anteriormente, relacionando las alturas y sombras, y escribe en la pizarra la proporción correspondiente. Un extracto de su relato se presenta a continuación.

PD1: Entonces nosotros, para ver cuáles son los lados proporcionales tenemos que ver qué es lo que podría ser proporcional con qué o qué podría ser semejante con qué, por ejemplo, la altura de la pirámide, ¿a qué lado de este triángulo va a ser proporcional o semejante?

Luego, asigna números a los catetos de cada triángulo y plantea la siguiente pregunta.

PD1: ¿Cómo determinamos si eso está en una proporción?

7. Según Duval (2005), una configuración nD/2D corresponde a las representaciones producidas en el plano (papel, pantalla de ordenador). 
Utilizando una propiedad de las proporciones, verifica si las razones entre los lados correspondientes son proporcionales (imagen 2), los números seleccionados se escogen aleatoriamente. PD1 no repara en la hipótesis del teorema en cuestión. El trabajo que originalmente se declaró como geométrico, cambia al dominio aritmético, donde el teorema de Thales es una herramienta para calcular un valor desconocido cuando dos razones están en proporción (lo llama "multiplicar cruzado»). El profesor desarrolla principalmente su trabajo, a través de tratamientos en el registro numérico (imagen 3).

Finalmente, el docente menciona que esto no solo sirve para casos con triángulos, sino también cuando las rectas son paralelas cortadas por dos transversales, idea que retoma en $t_{4}$. Luego, a modo de institucionalización, dibuja dos triángulos e indica qué lados son paralelos y escribe las proporciones entre los lados en lenguaje algebraico. El trabajo en $t_{2}$ finaliza con el siguiente relato.

PD1: Esa (recta) va a ser paralela a esa, porque es como si estuvieran las dos sobre el suelo. Son todos los casos de proporcionalidad que podríamos usar para este tipo de ejercicios.

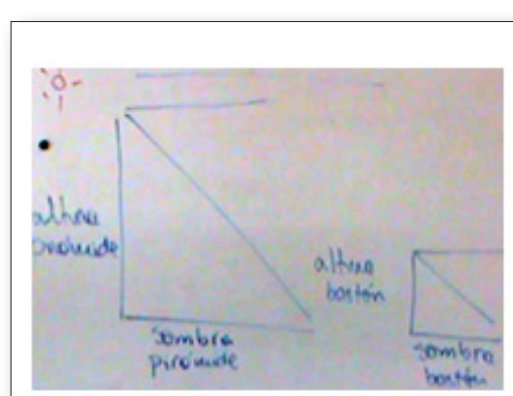

Imagen 1: triángulos semejantes

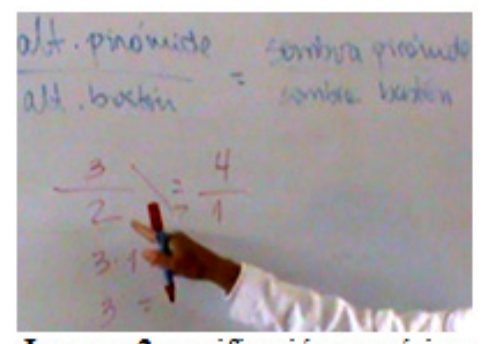

Imagen 2: verificación numérica

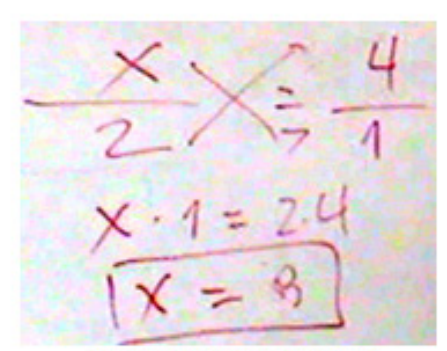

Imagen 3: calcular $x$

Fig. 2. Imágenes del trabajo de PD1.

\section{Circulación entre las componentes de los planos del ETM ${ }_{\mathrm{GS}}$}

La circulación entre las componentes de los planos del $\mathrm{ETM}_{\mathrm{GS}}$ de PD1 inicialmente es activada por la génesis figural, lo cual se realiza sobre la visualización de un tipo de configuración 2D/2D; la principal actividad sobre los triángulos representados en una posición particular fue relacionar aquellos atributos permiten decir que son figuras semejantes (ángulo recto del triángulo rectángulo y los catetos horizontal/ vertical). Aquí el proceso de visualización se relaciona con lo que Duval llama icónica, y específicamente del tipo botaniste. $\mathrm{Al}$ asignar números a los catetos de los triángulos (que se asumen rectángulos) y luego plantear una proporción, el trabajo activa un tipo de génesis instrumental, en el cual una propiedad es usada como artefacto simbólico que permite calcular un valor desconocido mediante tratamientos en el registro numérico (y luego artimético-algebraico). De este modo, observamos que el trabajo inicialmente está basado en la visualización, utilizando un tipo de figura que permite interpretar una proporción, y luego, sobre el empleo de una propiedad como un artefacto simbólico para calcular un valor desconocido.

Este espacio de trabajo dado a partir de $t_{2}$, que inicialmente fue declarado por el profesor en geometría, cambia a un dominio aritmético-algebraico, esto después de interpretadas las proporciones a partir de la activación de la génesis figural por la visualización icónica de las figuras. Posteriormente, el trabajo se basa en calcular un valor en registro numérico (similar en el caso de $t_{3}$ donde el trabajo se funda en la ejercitación algebraica), que es caracterizado por activar la génesis instrumental.

En lo que sigue de la clase, la circulación del espacio de trabajo analizado se repite centrando la atención en el uso de un tipo de herramientas, a partir de la visualización de ciertos ejemplos prototípicos, en referencia a Hershkowitz (como se cita en Scaglia y Moriena, 2005), y que promueven los tratamientos algebraicos y producción de resultados numéricos. 


\section{Paradigma Geométrico}

A nivel del Paradigma Geométrico privilegiado en la clase, analizamos el rol que cumple el teorema enseñado en el espacio de trabajo desarrollado por PD1. Como fue señalado anteriormente, el episodio 2 se sustenta sobre la representación de configuraciones geométricas (a mano alzada), y posteriormente, la interpretación de sus relaciones a partir de sus características visibles. En este sentido, la construcción con artefacto material no parece ser un trabajo relevante para el docente, al igual que la hipótesis sobre el paralelismo involucrado, tampoco lo es la justificación del teorema ni un trabajo de tipo discursivo. La relevancia está en el uso del teorema como una nueva herramienta que favorece un trabajo de cálculo en un registro numérico inicialmente y, luego, un trabajo de tipo algebraico al resolver ecuaciones (a partir del segundo episodio de la clase). De este modo, el trabajo analizado de PD1 privilegia la geometría natural (GI), en el cual se favorecen ciertos ejemplos prototípicos de triángulos que permiten plantear y resolver ecuaciones.

El profesor institucionaliza el nuevo saber al finalizar el segundo episodio en la clase, formulando el teorema desde su origen en el referencial -lo cual podría vincularse al paradigma GII-. Sin embargo, no privilegia un razonamiento discursivo donde la validación y justificación del teorema resulta ser relevante, la hipótesis de paralelismo se pierde bajo el tratamiento algebraico al resolver ecuaciones, o bien, en el que se podrían precisar e interpretar aspectos no abordados en torno a las tareas desarrolladas. Luego, esto se ofrece como tipos de tareas que permiten calcular un valor desconocido interpretando ejemplos prototípicos y empleando una nueva herramienta no material.

\section{Segundo caso: ETM $_{\mathrm{GC}}$ idóneo de PD2}

Contextualización de la clase

La siguiente sesión fue desarrollada en junio del año 2012 y corresponde a primer año medio (14 años). El tema que declara PD2 es Traslación de figuras en el plano cartesiano; se trata de la primera sesión donde se estudian las isometrías en el sistema de coordenadas cartesianas. Según el currículo (MINEDUC, 2009) se relaciona con el eje geometría, en particular el Contenido Minimo Obligatorio 14 que establece «Notación y representación gráfica de vectores en el plano cartesiano y aplicación de la suma de vectores para describir traslaciones de figuras geométricas» (p. 183).

Los conocimientos previos de los estudiantes involucran en el enfoque sintético la aplicación de transformaciones isométricas empleando un software (Geogebra), esto fue desarrollado en sesiones anteriores.

Con respecto al ambiente y clima que genera PD2 durante la clase, se observan escasas posibilidades de indagación e interacción entre los estudiantes. Si bien los alumnos participan desarrollando tareas en la pizarra, esto se hace sin cuestionamientos por parte del resto del curso.

En la tabla 4 se muestran las tareas dadas por PD2 durante la clase y el episodio que caracteriza este instante de la tarea $(t)$. 
Tabla 4.

Tareas dadas por PD2 y selección de $t$ clave

\begin{tabular}{|c|c|c|}
\hline $\begin{array}{l}\text { Tema central de } \\
\text { la clase }\end{array}$ & Episodio de la clase & Tarea $(t)$ \\
\hline \multirow{4}{*}{$\begin{array}{l}\text { Traslación de } \\
\text { figuras en el } \\
\text { plano cartesiano }\end{array}$} & $\begin{array}{l}\text { 1. Recuerda } \\
\text { conocimientos previos }\end{array}$ & $\begin{array}{l}t_{1}: \text { Trasladar un punto según un vector en un plano } \\
\text { cuadriculado. }\end{array}$ \\
\hline & $\begin{array}{l}\text { 2. Trata traslación en el } \\
\text { plano cartesiano }\end{array}$ & $\begin{array}{l}t_{2}: \text { Trasladar gráficamente un punto según un vector } \\
\text { dado. }\end{array}$ \\
\hline & $\begin{array}{l}\text { 3. Realiza ejercitación } \\
\text { para determinar un } \\
\text { vector }\end{array}$ & $t_{3}:$ Calcular las coordenadas de un vector traslación. \\
\hline & $\begin{array}{l}\text { 4. Realiza ejercitación } \\
\text { sobre traslación }\end{array}$ & $\begin{array}{l}t_{4}: \text { Representar puntos en el plano cartesianoy, calcular } \\
\text { las coordenadas de las imágenes al trasladarlos según un } \\
\text { vector dado. }\end{array}$ \\
\hline
\end{tabular}

En este caso seleccionamos las tareas clave $t_{2}$ y $t_{3}$, pues ambas se desarrollan de forma alternada. Destacamos que aún cuando las tareas tratan sobre la misma traslación de puntos, estas se desarrollan como trabajos distintos, lo cual se describe a continuación.

\section{Descripción del trabajo en torno a tareas clave}

Las tareas seleccionadas son: Trasladar gráficamente un punto según un vector dado, y Calcular las coordenadas del vector traslación. En este caso, PD2 utiliza un programa (Geogebra) para representar un punto inicial y el vector traslación en el sistema de coordenadas cartesianas. En el segundo episodio, $t_{2}$ consiste en contar las unidades horizontales y verticales donde se desplaza el punto en un plano cuadriculado (véase imagen 4). Esto se repite para cinco casos que varían según el punto inicial y el vector dado. De forma alternada se desarrolla $t_{3}$, para los cinco movimientos realizados en $t_{2}$. La vista algebraica del programa no es utilizada. En la pizarra PD2 dibuja una tabla (imagen 5) y señala a los estudiantes que deben escribir las coordenadas del punto y de su imagen, luego, les solicita calcular las coordenadas del vector -representado gráficamente en $t_{2}-$.

En $t_{2}$ el énfasis está en la visualización de los puntos que se trasladan, mientras que en $t_{3}$ el trabajo es calcular mediante tratamientos numéricos las coordenadas del vector traslación; se realiza restando coordenadas entre dos puntos (punto inicial y final que representan al vector). En $t_{3}$, PD2 indica que deben llevar el vector al origen para determinar sus coordenadas. Para evitar errores PD2 señala lo siguiente.

PD2: Recordar que se debe mantener el orden porque si no, el vector no va a tener las coordenadas correspondientes y no vamos a poder interpretar bien la propiedad.

Los estudiantes participan desarrollando el trabajo en la pizarra. Una vez realizadas $t_{2}$ y $t_{3}$, con la intención de institucionalizar el trabajo, PD2 pregunta lo siguiente.

PD2: ¿Qué hacemos con las coordenadas para encontrar la imagen?

A continuación, para definir una traslación PD2 escribe: Una traslación $T_{\vec{v}}$ según un vector $\vec{v}\left(v_{1}, v_{2}\right)$ al punto $P(x, y)$ está dado por $T_{\vec{v}}(x, y)=\left(x+v_{1}, y+v_{2}\right)$. Lo anterior es usado en el siguiente episodio $(t)$ para calcular las coordenadas de puntos que se trasladan según un vector dado, y luego, graficarlos (imagen 6). 


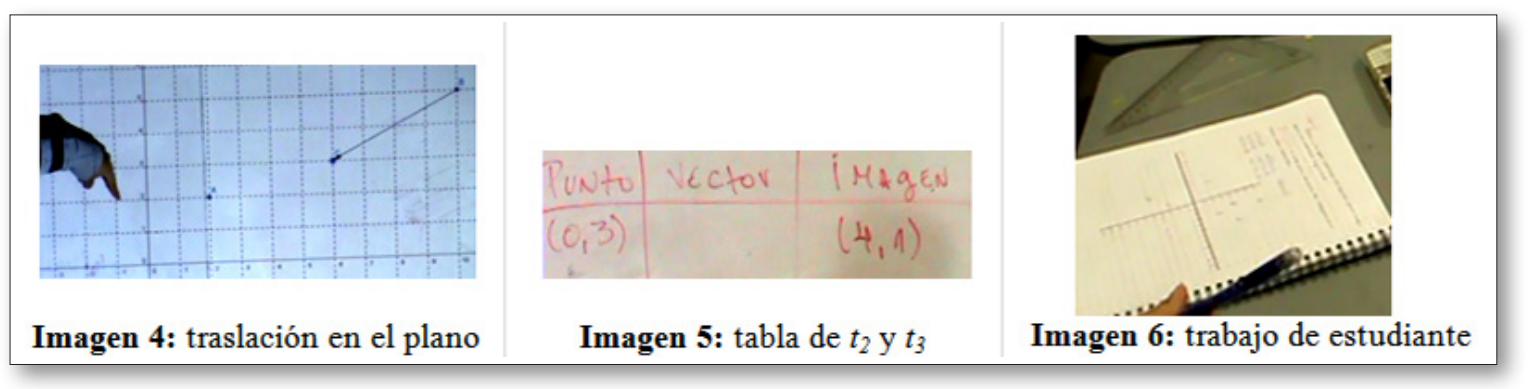

Fig. 3. Imágenes del trabajo de PD2

\section{Circulación entre las componentes de los planos del $\mathrm{ETM}_{\mathrm{GC}}$}

En los dos episodios descritos, $t_{2}$ y $t_{3}$ se van desarrollando alternadamente en cinco oportunidades. En términos de circulación, la traslación en el plano no se justifica ni se coordina con los tratamientos numéricos para obtener un vector (y viceversa). Esto provoca que ambas tareas se desarrollen desarticuladas entre sí.

En el primer caso, $t_{2}$ se desarrolla activando la génesis figural, en el cual la visualización para trasladar y localizar los puntos es el trabajo principal. En $t_{3}$ el trabajo consiste en obtener coordenadas, activando la génesis instrumental. Resaltamos que ambos trabajos en torno a dichas génesis no se conectan entre sí; lo desarrollado en $t_{2}$ no se coordina con $t_{3}$, aunque se trata de los mismos objetos involucrados (componente real y local del $\mathrm{ETM}_{\mathrm{G}}$ ).

En el espacio de trabajo activado por $t_{2}$, la visualización se desarrolla de modo icónico, pues la imagen se obtiene contando las unidades a la derecha o izquierda, y hacia arriba o abajo, en un cuadriculado según las características que se observan del vector dado. En el trabajo que activa $t_{3}$ se utiliza un tipo de artefacto simbólico para calcular las coordenadas del vector.

En general, PD2 no justifica el trabajo ni lo promueve en sus estudiantes, en relación a que las propiedades invariantes de la traslación no aparecen en su trabajo. Aun cuando en $t_{3}$ el profesor determina las coordenadas del vector traslación mediante un trabajo de cálculo numérico, no considera la suma de vectores para describir dicha trasformación (señalado en el currículo). De este modo, la génesis discursiva no es parte importante del trabajo de PD2 en torno a dichas tareas, tampoco lo es la génesis instrumental en relación al artefacto tecnológico que emplea, desaprovechando la vista algebraica que este proporciona y su potencial dinámico, pues entre otras, podría coordinar la visualización dinámica y la génesis discursiva al conjeturar respecto de las propiedades geométricas de las transformaciones realizadas.

Al finalizar el tercer episodio, PD2 se dispone a dar un carácter formal al trabajo realizado, definiendo (algebraicamente) el movimiento en términos de las coordenadas de un punto $P$ y su imagen. En términos de prueba en el ETM $\mathrm{E}_{\mathrm{G}}$, PD2 valida lo anterior a partir de los cinco ejemplos registrados en la tabla (imagen 5), lo cual constituye una prueba del tipo empirismo ingenuo. En $t_{4}$ el procedimiento definido algebraicamente es usado como un artefacto simbólico para calcular coordenadas.

\section{Paradigma Geométrico}

Sobre el paradigma geométrico privilegiado analizamos el rol de la traslación al ser enseñada por PD2. Inicialmente, para trasladar cuenta las unidades que se desplaza el punto según el vector dado gráficamente, privilegiando la percepción. Aquí, el modelo geométrico (cartesiano) está directamente relacionado al mundo físico (o real). Luego, respecto al trabajo que permitió obtener las coordenadas del vector, este fue desarrollado mediante un procedimiento de cálculo numérico, una vez determinadas las coordenadas de dos puntos dados gráficamente. Si bien al inicio el trabajo se activa por la 
génesis figural, esto no es articulado con el trabajo de cálculo que se caracteriza por privilegiar la génesis instrumental, en el cual se usa una operación aritmética para resolver la tarea. Lo anterior, no se justifica ni se sustenta en elementos del referencial que provienen de la transformación del plano estudiado y su definición en un sentido más formal.

Por las razones expuestas, el trabajo del profesor (PD2) privilegia el paradigma geometría natural (GI), donde se favorece una visualización icónica de los objetos involucrados y un tipo de artefacto simbólico para el cálculo numérico que permite determinar coordenadas. En el discurso y desarrollo del trabajo, PD2 no contempla ni promueve la génesis discursiva de la prueba.

\section{PERSPECTIVAS PARA ABORDAR LA COMPLEMENTARIEDAD ENTRE LOS ENFOQUES SINTÉTICO Y ANALÍTICO}

Los análisis de la práctica en el aula de los profesores analizados reflejan una dinámica sobre el espacio de trabajo geométrico idóneo. Esto nos permite estudiar en detalle procesos cognitivos, aspectos matemáticos, y cómo estos se relacionan en el trabajo realizado por un profesor en su quehacer en el aula. Destacamos la importancia de la tarea como activadora del trabajo, así como el paradigma privilegiado por cada profesor, y nuestro interés en abordar el tema de la complementariedad entre los enfoques geométricos sintético y analítico. A continuación, proponemos perspectivas según dicho propósito a partir de los ETM analizados.

En el caso del trabajo realizado por PD1 sobre el teorema de Thales, observamos que está basado en resolver una ecuación; los signos empleados (la equis) no son interpretadas desde el dominio geométrico, lo que reduce a que se desarrolle una "geometría algebrizada». Desde este punto de vista, el rol del profesor es fundamental para que potencie el ETM que se desea desarrollar y no uno que se reduzca a la «algebrización» (Montoya-Delgadillo, Mena-Lorca y Mena-Lorca, 2014). Para ilustrar la forma de trabajar del profesor, presentamos un extracto de la propuesta de texto escolar vigente (en relación con la clase observada); las tareas privilegian ejemplos prototípicos y los cálculos en dominio aritméticoalgebraico luego de interpretar las proporciones, tal como se muestra en la figura 4.

4. Determina el valor de $x$ de manera que se cumpla $R T / / P Q$.

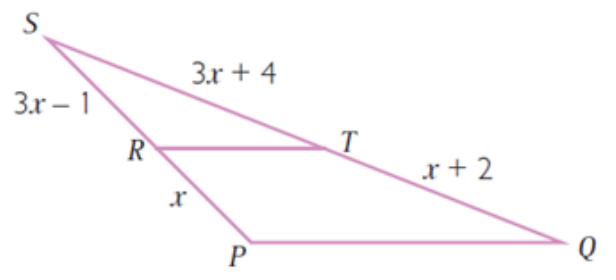

Fig. 4. Tarea propuesta en el texto escolar de segundo medio (Zañartu, Darrigrandi, y Ramos. 2012:161).

En relación al trabajo geométrico sintético desarrollado por PD1, y el tipo de tareas propuestas en el texto, observamos que inicialmente interviene una figura que permite interpretar proporciones, que luego se resuelven mediante tratamientos numéricos o algebraicos. Resaltamos que en este tipo de trabajo sintético se podría favorecer el tránsito al enfoque analítico, especialmente en aquellos que involucran tratamientos en los registros numérico y algebraico (asociados a teoremas como el de Thales o de Pitágoras). En general, el uso de signos y la semiótica intencionada favorecería el tránsito, especialmente, aquellos sobre tratamientos y conversiones entre los registros numérico y algebraico, tomando en cuenta la perspectiva cognitiva de Duval (1995). 
No obstante lo anterior, se debe poner atención, pues si bien el trabajo podría contribuir, se debe reflexionar sobre los riesgos de caer en los cálculos ciegos al resolver ecuaciones, perdiendo el sentido del trabajo geométrico en sí y provocando un cambio de dominio, de geométrico a aritmético/algebraico, sin retorno al dominio inicial (Montoya-Delgadillo y Vivier, 2014), como es el caso analizado de la práctica en el aula de PD1.

$\mathrm{Al}$ referirnos al trabajo desarrollado por PD2, aludimos al currículo escolar (véase sección sobre el hábitat de la geometría en secundaria). En relación a los contenidos relacionados con las isometrías, hemos constatado que es escasa la información proporcionada al profesor sobre cómo organizar la enseñanza o proporcionar ejemplos pertinentes, entre otros aspectos que no aparecen explícitamente definidos. En el nivel 8 (13 años), anterior al de la clase de PD2, según el currículo se estudian los movimientos rígidos del plano desde el enfoque sintético, y se evoca la idea algebraica de grupo y de las propiedades invariantes. En el nivel siguiente (1. ${ }^{\circ}$ medio, 14 años), el currículo presenta las isometrías en el enfoque analítico. Sin embargo, falta información que oriente el trabajo del docente, y que favorezca la coordinación entre ambos enfoques. Consideramos que los enfoques deben tratarse en continuidad, y las limitaciones del enfoque sintético se pueden retomar en el enfoque analítico, así se profundiza al enseñar los objetos geométricos.

Para favorecer la complementariedad entre ambos enfoques desde el ETM, consideramos que se debería promover la activación de la génesis discursiva y la componente referencial del plano epistemológico, pues según los análisis evidenciamos que el uso de artefactos simbólicos no se justifican, y se realizan débiles razonamientos discursivos entorno a la prueba. Esta situación se observó en particular en PD1 y PD2, pero también en los otros PD analizados del estudio. Suponemos que esto podría deberse, en gran medida, a que el profesor desconoce cómo realizar transposiciones adecuadas, en particular en el proceso de prueba, pues el profesor novel no es sometido a una reflexión explícita de la transposición de la demostración o de otro tipo de argumentación en su formación inicial (MontoyaDelgadillo, 2014). O bien, orientaciones sobre qué tareas proponer para activar un $\mathrm{ETM}_{\mathrm{G}}$ personal más favorable en sus estudiantes.

Este es un desafío a continuar. El paso siguiente en esta investigación, es ofrecer situaciones de referencia como un material de trabajo para profesores y para la formación inicial.

\section{CONCLUSIONES}

Por un lado, al retomar las preguntas planteadas inicialmente sobre la práctica en el aula de profesores en relación a temáticas geométricas, cuando los enfoques son sintéticos o analíticos, resaltamos la relevancia y pertinencia de usar una herramienta de análisis como es el marco del ETM, pues nos permite identificar con detalle cómo se articulan los procesos cognitivos y los elementos matemáticos en el trabajo (en este caso) del profesor, las circulaciones que privilegia, o las génesis puestas (o no) en juego. Esto nos permitió observar que en ambos ETM $_{\mathrm{G}}$ idóneos estudiados el enfoque sintético se desarrolla desconectado del analítico, incluso cuando las temáticas lo permiten. Reconocemos que el ETM idóneo del docente varía según los conocimientos de sus estudiantes, según lo que demanda la institución, los objetivos de la enseñanza, entre otros. Luego, cabe precisar que una clase no es suficiente para afirmar el paradigma privilegiado o desarrollado por el profesor, sino que un análisis en su conjunto, más el uso de otros insumos (entrevistas, uso de textos, guías de ejercicios, etc.), nos permitirían acceder al paradigma geométrico desplegado. No obstante lo anterior, los resultados presentados permiten mostrar en profundidad los análisis de la práctica en el aula del profesor en relación con objetos matemáticos específicos. 
Por otro lado, este análisis nos permite reconocer dificultades en el interior de cada enfoque geométrico y la posibilidad de plantear perspectivas para abordar la problemática en cuestión. En particular, consideramos que para favorecer el tránsito y la complementariedad entre los enfoques geométricos es necesario enriquecer el trabajo en cada enfoque con problemas que activen las tres génesis y así permita al estudiante un trabajo cognitivo favorable. Además, sería pertinente analizar en una secuencia de aprendizaje qué circulaciones son las que se pretenden favorecer y las que se ven obstaculizadas para enriquecer el desarrollo del ETM del estudiante.

Para profundizar en el trabajo matemático de los profesores analizados, hemos considerado una metodología sustentada en el ETM que se basa en el estudio de tareas propuestas por el profesor (que son las activadoras del ETM) y de las circulaciones entre las componentes de los planos del espacio de trabajo matemático. Además, fueron considerados elementos del currículo de secundaria, los estándares para la formación de profesores y un texto escolar, que contribuyen a contextualizar y complementar los análisis. El currículo y los estándares para la formación de profesores plantean la intención de dar continuidad a los enfoques geométricos; sin embargo, no aportan información al docente respecto a cómo puede hacerlo. En cuanto al texto escolar, la propuesta en torno al teorema de Thales privilegia los tratamientos numérico/algebraicos, situación que no es particular del texto seleccionado.

En el caso de PD1, que emplea métodos sintéticos, el trabajo que inicialmente se declara como geométrico y se inicia desde la génesis figural se transforma en un trabajo que privilegia lo aritméticoalgebraico, donde el teorema de Thales pasa a segundo plano y se usa una propiedad como un artefacto simbólico para permitir resolver ecuaciones. En este espacio de trabajo, los artefactos materiales para realizar construcciones geométricas semejantes no se emplean y las justificaciones en el trabajo discursivo del dominio geométrico son escasamente tomadas en consideración. Con esto, no queremos afirmar que no se realicen técnicas algebraicas, sino al contrario, el punto es resaltar la importancia del álgebra en el ETM en distintos dominios, y teniendo esto en consideración el trabajo de los docentes debería aprovechar estas técnicas para desarrollar un ETM pertinente para sus estudiantes.

En el caso de PD2, que trabaja con métodos cartesianos, la activación de las génesis se desarrolla del mismo modo que en PD1. Es decir, el trabajo es activado desde la génesis figural cuando se mueve un punto según las características físicas de un vector y, luego, la génesis instrumental, empleando un tipo de herramienta simbólica que permite determinar coordenadas de un vector. Esta forma de trabajar se desarrolla desarticulada entre sí, aunque se trata de la propia traslación de puntos, y si bien se desarrolla en un entorno tecnológico, los desplazamientos y la vista algebraica que proporciona el programa no son explotados en su potencial.

En ambos casos analizados, los profesores relegan del trabajo la génesis discursiva de la prueba (o no es la que se privilegia); consideran débilmente razonamientos argumentativos sustentados en elementos de la componente referencial. Es decir, las justificaciones y validaciones de los procedimientos desarrollados no se fundamentan en propiedades y definiciones que favorecen los razonamientos de validación. Por otro lado, la génesis semiótica y la génesis instrumental fueron privilegiadas por PD1 y PD2 (y por el resto de PD) en este estudio. En términos teóricos, ambos trabajos analizados circulan y privilegian el plano vertical Sem-Ins. ${ }^{8}$ En el caso de PD1, el trabajo en el dominio geométrico (sintético) cambia a otro dominio sin retorno al dominio original, y si bien los tratamientos algebraicos podrían contribuir a transitar a la geometría analítica, esto no es evidente en el trabajo del profesor. En el trabajo de PD2, las génesis que se activan no son articuladas. En ambos casos (PD1, PD2), la génesis instrumental se centra en el uso de un tipo de artefacto simbólico para dar solución al problema. Se debe destacar que al finalizar la clase ambos profesores formalizan los temas tratados. Esto permite

8. Sobre los planos verticales, se pueden consultar trabajos recientes en el marco del ETM (Coutat y Richard, 2011; Kuzniak y Richard, 2014). 
inferir que en este escenario resulta difícil pensar en tránsito y complementariedad entre los enfoques geométricos.

Finalmente, destacamos que incluso cuando el propósito de este estudio no es generalizar respecto al $\mathrm{ETM}_{\mathrm{G}}$ idóneo del profesor, los resultados aportan información relevante respecto al trabajo geométrico sintético y analítico en el nivel secundario. Además, presentamos un análisis del trabajo del profesor usando el ETM como una herramienta teórica para su estudio. Este análisis contribuye al diseño de situaciones de referencia que favorecen el tránsito y complementariedad entre los enfoques, que serán un material de trabajo para los profesores de secundaria y para su formación inicial.

\section{AGRADECIMIENTOS}

Esta investigación ha sido parcialmente financiada por una beca doctoral de la Pontificia Universidad Católica de Valparaíso, y por el proyecto Fondecyt 1110988.

\section{REFERENCIAS BIBLIOGRÁFICAS}

Artigue, M. (2002). Learning mathematics in a CAS environment: the genesis of a reflection about instrumentation and dialectics between technical and conceptual work. International Journal of Computer for Mathematical Learning, 7(3), pp. 245-274.

http://dx.doi.org/10.1023/A:1022103903080

Badillo, E.; Figueiras, L.; Font, V. y Martínez, M. (2013). Visualización gráfica y análisis comparativo de la práctica matemática en el aula. Enseñanza de las Ciencias, 31(3), pp. 207-225.

Balacheff, N. (1987). Processus de preuve et situations de validation. Educational Studies in Mathematics, 18(2), pp. 147-176.

http://dx.doi.org/10.1007/BF00314724

Bishop, A.J. (1987). Quelques obstacles à l'apprentissage de la géométrie. En R. Morris (ed.). Études sur l'enseignement des mathématiques: L'enseignement de la géométrie vol. 5. Paris, France: Unesco, pp. 149-169.

Bourbaki, N. (1969). Formas cuadráticas. Geometría elemental. En J. Hernández (ed.). Elementos de historia de las matemáticas. Madrid, España: Alianza Editorial, pp. 173-191.

Centro de Perfeccionamiento, experimentación e investigaciones pedagógicas (2008). Marco para la Buena Enseñanza. Santiago, Chile: Ministerio de Educación.

Centro de Perfeccionamiento, experimentación e investigaciones pedagógicas (2012). Estándares disciplinarios para la enseñanza: Matemática. En Estándares Orientadores para Carreras de Pedagogía en Educación Media. Santiago, Chile: Ministerio de Educación, pp. 89-132.

Chevallard, Y. (1999). L'analyse des pratiques enseignantes en théorie anthropologique du didactique. Recherches en Didactique des Mathématiques, 19(2), pp. 221-266.

Coutat, S. y Richard, P. (2011). Les figures dynamiques dans un espace de travail mathématique pour l'apprentissage des propriétes géométriques. Annales de Didactique et de Sciences Cognitives, 16, pp. 97-126.

De Villiers, M. (1993). Transformations: A golden thread in school mathematics. Disponible en línea: <http://mzone.mweb.co.za/residents/profmd/transform.pdf>.

Dieudonné, J. (1964). Prólogo del libro Álgebra lineal y geometría elemental. En J. Hernández (ed.). La Enseñanza de las Matemáticas Modernas. Madrid, España: Alianza Editorial, pp. 271-284.

Duval, R. (1995). Sémiosis et pensée humaine. Registres sémiotiques et apprentissages intellectuels. Berne, Suisse: Peter Lang. 
Duval, R. (1999). Representation, vision and visualization: Cognitive functions in mathematical thinking. Basic issues for learning. En F. Hitt y M. Santos (eds.). Proceedings of the 21st North American PME Conference, 1, pp. 3-26.

Duval, R. (2005). Les Conditions Cognitives de l'apprentissage de la géométrie: Développement de la Visualisation, Différenciation des Raisonnements et Coordination de leurs Fonctionnements. Annales de Didactique et de Sciences Cognitives, 10, pp. 5-53.

Gascón, J. (2002). Geometría sintética en la ESO y analítica en el bachillerato. ¿Dos mundos completamente separados? Suma, 39, pp. 13-25.

Gascón, J. (2003). Efectos del autismo temático sobre el estudio de la geometría en secundaria. Suma, 44 , pp. 25-34.

Houdement, C. y Kuzniak, A. (1996). Autours des stratégies utilisées pour former les maitres du premier degré en mathématiques. Recherches en Didactique des Mathématiques, 16(3), pp. 283-312.

Houdement, C. y Kuzniak, A. (1999). Un exemple de cadre conceptuel pour de l'enseignement de la géométrie en formation des maîtres. Educational Studies in Mathematics, 40, pp. 283-312. http://dx.doi.org/10.1023/A:1003851228212

Houdement, C. \& Kuzniak, A. (2006). Paradigmes géométriques et enseignement de la géométrie. Annales de Didactique et de Sciences Cognitives, 11, pp. 175-193.

KLeIn, F. (1908). Matemática elemental desde un punto de vista superior: Geometría vol. 2. Traducción de R. Fontanilla. Madrid, España: Biblioteca Matemática.

Kuzniak, A. (2011). L'Espace de Travail Mathématique et ses Genèses. Annales de Didactique et de Sciences Cognitives, 16, pp. 9-24.

KuzniaK, A. \& Richard, P. (2014). Espaces de travail mathématique. Points de vue et perspectives. Revista Latinoamericana de Investigación en Matemática Educativa (Número Especial).

Ministerio de Educación. (2009). Matemática: Formación general. En Objetivos Fundamentales y Contenidos Minimos Obligatorios de la Educación Básica y Media. Santiago, Chile: Autor, pp. 145-194.

Montoya-Delgadillo, E. (2014). El proceso de prueba en el espacio de trabajo geométrico: profesores en formación inicial. Revista Enseñanza de las Ciencias, 32(3), pp. 227-247.

Montoya-Delgadillo, E.; Mena-Lorca, A. y Mena-Lorca, J. (2014). Circulaciones y génesis en el espacio de trabajo matemático. Revista Latinoamericana de Investigación en Matemática Educativa, 17(4-I), pp. 191-210 (en prensa).

Montoya-Delgadillo, E. \& Vivier, L. (2014). Les changements de domaine de travail dans le cadre des Espaces de Travail Mathématique. Annales de Didactique et de Sciences Cognitives, 19, pp. 73-101. http://dx.doi.org/10.5565/rev/ensciencias. 1049

Organización de las Naciones Unidas para la Educación, la Ciencia y la Cultura (1973). Nuevas Tendencias en la Enseñanza de la Matemática, vol. 3. París, Francia: Autor.

Peirce, C. (1978). Ecrits sur le signe. Paris, France: Seuil.

Rabardel, P. (1995). Les Hommes et les Technologies: Une approche cognitive des instruments contemporains. Paris, France: Armand Colin.

Santaló, L. (1980). Situación de la enseñanza de la geometría frente a las nuevas tendencias de la educación matemática. Revista de Bachillerato, suplemento del n. ${ }^{\circ}$ 13, pp. 23-28.

Scaglia, S. y Moriena, S. (2005). Prototipos y estereotipos en geometría. Educación Matemática, 17(3), pp. 105-120.

Trouche, L. (2002). Une approche instrumentale de l'apprentissage des mathématiques dans des environnements de calculatrice symbolique. En D. Guin y L. Trouche (eds.). Calculatrices Symboliques. Transformer un outil du travail informatique: un problème didactique. Grenoble, France: La Pensée Sauvage, pp. 187-214.

Zañartu, M.; Darrigrandi, F. y Ramos, M. (2012). Matemática 2. Año Medio: Texto para el Estudiante. Santiago, Chile: Santillana. 


\title{
Synthetic and analytic geometrical working space of teachers and their practices in the classroom
}

\author{
Carolina Henríquez Rivas, Elizabeth Montoya Delgadillo \\ Instituto de Matemáticas. Pontificia Universidad Católica de Valparaiso, Chile \\ carohenriquezrivas@gmail.com, emontoya@ucv.cl
}

This article refers to a current problem related to ways of teaching geometry at secondary level, the complementarity between the approaches of Euclidean geometry with and without Cartesian coordinates -where the names analytical geometry and synthetic geometry are used. To differ between such approaches, we base on the distinction offered by Klein (1908): 'in synthetic geometry, figures are studied without the intervention of formulae, and in the analytical geometry, these are applied by means of the use of the coordinates system'.

In the famous Program of Erlangen, Klein proposes the cessation to the disputes between synthetic and analytical geometry, so under this point of view, "geometry» would be the set of invariant properties by means of the transformations of the corresponding group (Bourbaki, 1969). The teaching of geometry is a matter that has been approached by diverse authors for more than a century, not only by mathematicians, but also by didactics of the mathematics leading to controversies that periodically have been restated (Dieudonné, 1964; Bishop, 1987; Santaló, 1980; De Villiers, 1993; Gascón, 2002, 2003).

In this investigation the notion of transit is understood as the change from synthetic approach to the analytical one (or vice versa); and the notion of complementarity refers to the idea of influence with each other, which on one hand leads to a deep understanding of the topics covered in the school, and on the other hand to the possibility of linking geometry with other fields of mathematics.

We suggest a qualitative study on two novice teachers' work in the classroom ${ }^{1}(\mathrm{PD})$ of secondary education during the second semester of 2011 and the first semester of 2012, recording 10 (or more) lessons. Their initial training took place in different Chilean universities. In general, the requirements to become a secondary teacher in Chile consist of 5 years in full time education, and the course considers modules of mathematics, mathematical education and professional practices -the emphasis changes depending on the institution.

To penetrate into the mathematical work of teachers in their classroom practice from a specific theoretical model, and particularly on subject matters of synthetic and analytical geometry, we have considered the theory of Mathematical Working Space, MWS ${ }^{2}$ Kuzniak, 2011). We are interested in knowing how epistemological and cognitive aspects are revealed in secondary teachers' practices, when the subject matters develop according to each geometric approach.

The analyses developed in both ideal $\mathrm{MWS}_{\mathrm{G}}$ (ideal $\mathrm{MWS}_{\mathrm{G}}$ in Spanish: Espacio de Trabajo Geométrico idóneo) concluded that the synthetic approach develops disconnected from the analytical one, still when the subject matters allow it. In both of the cases studied, the discursive genesis of the proof has been relegated by teachers (which is not exclusively used), and argumentative reasoning based on referential elements is weakly considered. That is to say, the justifications and validations of the developed procedures are not based on properties and definitions that act in favor of the reasoning of validations. On the other hand, in precise terms of the MWS, the semiotic genesis and the instrumental genesis were favored by both novice teachers (PD) who appear in this study, and also for the rest of the PD participants (6 in total).

1. Teachers with up to 3 years of professional experience. We focus on these teachers, since this work subscribes in a wider investigation on the novice teachers ETM in Chile.

2. MWS in Spanish: Espacio de Trabajo Matemático. 